\title{
SEMIGROUPS OF MULTIPLIERS ASSOCIATED WITH SEMIGROUPS OF OPERATORS
}

\section{A. OLUBUMMO}

ABSTRACT. Let $G$ be an infinite compact group with dual object $\Sigma$. Corresponding to each semigroup $\mathfrak{J}=\{T(\xi) ; \xi \geq 0\}$ of operators on $L_{p}(G), 1 \leq p<\infty$, which commutes with right translations, there is a semigroup $\mathcal{E}=\left\{E_{\xi}(\sigma) ; \xi \geq 0, \sigma \in \Sigma\right\}$ of $L_{p}(G)$ multipliers. If $\zeta$ is strongly continuous, then $\left\{E_{\xi}(\sigma) ; \xi \geq 0\right\}$ is uniformly continuous for each $\sigma$. Conversely a semigroup $\mathscr{E}$ of $L_{p}(G)$-multipliers determines a semigroup $\Im$ of operators on $L_{p}(G)$. $\Im$ is strongly continuous if each $E_{\xi}(\sigma)$ is uniformly continuous; and then there exist a function $A$ on $\Sigma$ and $\Sigma_{0} \subset \boldsymbol{\Sigma}$ such that $E_{\xi}(\sigma)=E_{0}(\sigma) \exp \left(\xi A_{\sigma}\right)$ if $\sigma \epsilon \Sigma_{0}$ and $E_{\xi}(\sigma)=0$ if $\sigma \notin \Sigma_{0}$.

1. Introduction. Let $X$ be a Banach space and denote by $B(X)$ the Banach algebra of all bounded linear operators on $X$ with the operator norm. A family $\mathcal{T}=\{T(\xi) ; \xi \geq 0\}$ of operators in $B(X)$ is called a strongly continuous semigroup of operators on $X$ if

(i) $T\left(\xi_{1}+\xi_{2}\right) x=T\left(\xi_{1}\right)\left[T\left(\xi_{2}\right) x\right], \xi_{1}, \xi_{2} \in[0, \infty), x \in X$;

(ii) $\lim _{\xi \rightarrow 0^{+}} T(\xi) x=T(0) x, x \in X$.

If (i) holds and (ii) is replaced by

(iii) $\lim _{\xi \rightarrow 0^{+}}\|T(\xi)-T(0)\|=0$, then $\mathcal{J}$ is called a uniformly continuous semigroup of operators on $X$.

Let $G$ be an infinite compact group with dual object $\Sigma$. We denote by $\checkmark(\Sigma)$ the set $P B_{\sigma \in \Sigma}\left(H_{\sigma}\right)$, where $H_{\sigma}$ is the representation space of the representation $U^{(\sigma)}[1,28.24]$. Suppose that $\mathcal{Q}$ and $B$ are subsets of $\widetilde{N}(\Sigma)$. An element $E$ of $(\Sigma(\Sigma)$ is said to be an (Ql, B)-multiplier if $E A \in \mathbb{B}$ for all $A \in \mathbb{Q}[1,35.1]$. If $E$ is an ( $(2$, Il)-multiplier, we shall call $E$, simply, an \&l-multiplier.

Throughout this paper, $G$ denotes an infinite, compact group with dual object $\Sigma$. Haar measure on $G$ is denoted by $\lambda$, and it will be assumed that $\lambda$ is normalized so that $\lambda(G)=1$. For $1 \leq p<\infty, L_{p}(G)$ denotes the usual Lebesgue space formed with respect to $\lambda$. The set of Fourier transforms $\hat{f}$

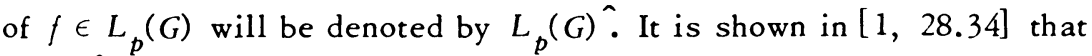
$L_{p}(G)^{\wedge}$ is a subset of $(S(\Sigma)$. To simplify our notation, we shall write ' $L_{p}(G)$-multiplier' in place of ' $L_{b}(G)$-multiplier'.

Received by the editors November 5, 1973.

AMS (MOS) subject classifications (1970). Primary 43A22, 43A30, 47D05. 
By a semigroup of $L_{p}(G)$-multipliers we shall mean a function $E$ on $[0, \infty) \times \Sigma$ such that

(i) for each pair $(\xi, \sigma), E_{\xi}(\sigma) \in B\left(H_{\sigma}\right)$;

(ii) for each fixed $\xi, E_{\xi}(\cdot)$ is an $L_{p}(G)$-multiplier;

(iii) for each fixed $\sigma,\left\{E_{\xi}(\sigma) ; \xi \geq 0\right\}$ is a semigroup of operators on $H_{\sigma}$.

The results of this paper can be summarized as follows. Given a semigroup $\mathcal{T}=\{T(\xi) ; \xi \geq 0\}$ of operators on $L_{p}(G)$, the elements of which commute with right translations, we associate with $\mathcal{T}$ a semigroup $\mathscr{E}=$ $\left\{E_{\xi}(\sigma) ; \xi \geq 0, \sigma \in \Sigma\right\}$ of $L_{p}(G)$-multipliers. We show that if $\mathcal{T}$ is strongly continuous, then $\left\{E_{\xi}(\sigma) ; \xi \geq 0\right\}$ is uniformly continuous for each $\sigma$. Conversely, given a semigroup $\mathcal{E}=\left\{E_{\xi}(\sigma) ; \xi \geq 0, \sigma \in \Sigma\right\}$ of $L_{p}(G)$-multipliers, we associate with $\mathcal{E}$ a semigroup $\mathscr{T}=\{T(\xi) ; \xi \geq 0\}$ of operators on $L_{p}(G)$, the members of which commute with right translations. We prove, moreover, that if $\left\{E_{\xi}(\sigma) ; \xi \geq 0\right\}$ is uniformly continuous for each $\sigma$, then $\mathcal{T}$ is strongly continuous. Furthermore, we show that there exist $A=\left(A_{\sigma}\right)$ in $(\mathcal{S}(\Sigma)$ and a subset $\Sigma_{0}$ of $\Sigma$ such that $E_{\xi}(\sigma)=E_{0}(\sigma) \exp \left(\xi A_{\sigma}\right)$ if $\sigma \in \Sigma_{0}$ and $E_{\xi}(\sigma)$ $=0$ if $\sigma \notin \Sigma_{0}$. Finally, we prove that if $Q$ is the infinitesimal operator of $\mathcal{T}$, then $A$ is a $\left(D(\mathfrak{A}), L_{p}(G)\right)$-multiplier.

The results and proofs in the present paper generalize to arbitrary infinite compact groups those of Hille [2, Theorems 20.3.1 and 20.3.2] for the circle group and those obtained in [5] for compact Abelian groups. It will be clear, however, that the orientation here is somewhat different from that of [2] and [5]. Moreover, it is hoped that our proofs and results shed some light on the classical situation.

2. Preliminaries. Let $G$ and $\Sigma$ be as defined above. It will be assumed throughout this paper that, for each $\sigma \in \Sigma$, a fixed representation $U^{(\sigma)}$ with representation space $H_{\sigma}$ has been chosen and that, in each $H_{\sigma}$, a fixed conjugation $D_{\sigma}$ has been chosen. It will be understood that all Fourier-Stieltjes transforms and Fourier transforms are defined in terms of these fixed $U^{(\sigma) \text { 's }}$ and $D_{\sigma}$ 's. In this and other definitions and notation, we follow Hewitt and Ross [1] where any undefined terms concerning harmonic analysis, used in this paper, will be found. Similarly, the reader is referred to Hille and Phillips [2] for an account of the theory of semigroups of operators on a Banach space.

2.1. Lemma. Let $\sigma \in \Sigma$ and for $U^{(\sigma)}$ in $\sigma$ with representation space $H_{\sigma}$ let $\mathfrak{T}_{\sigma}(G)$ denote the set of all finite complex linear combinations of functions of the form $x \rightarrow\left\langle U_{x}^{(\sigma)} \xi, \eta\right\rangle$ as $\xi, \eta$ vary over $H_{\sigma}$. Then $\{\hat{f}(\sigma): f \in$ $\left.\mathfrak{T}_{\sigma}(G)\right\}=B\left(H_{\sigma}\right)$. 
This is [1, Theorem (28.39)(i)].

2.2. Lemma. Let $T$ be a bounded linear operator on $L_{p}(G)$ which commutes with right translations. Then there exists a unique $E \in \mathbb{S}(\Sigma)$ such that $\left(T f \hat{)}(\sigma)=E(\sigma) \hat{f}(\sigma)\right.$ for all $f \in L_{p}(G)$ and all $\sigma \in \Sigma$.

Proof. Since $T$ commutes with right translations a routine argument shows that $T(f * g)=(T f) * g$ for all $f, g \in L_{p}(G)$ (see e.g. [1, p. 376]). The result now follows from Theorem 35.8 of [1] and Lemma 2.1 above.

\section{Semigroups of operators and semigroups of multipliers.}

3.1. Theorem. Let $\mathfrak{T}=\{T(\xi) ; \xi \geq 0\}$ be a semigroup of bounded linear operators on $L_{p}(G)$, each of which commutes with right translations. Then $\mathcal{T}$ defines a semigroup $\mathcal{E}=\left\{E_{\xi}(\sigma) ; \xi \geq 0, \sigma \in \Sigma\right\}$ of $L_{p}(G)$-multipliers. If, in addition, $\mathcal{T}$ is strongly continuous, then, for each $\sigma \in \Sigma$, the set $\left\{E_{\xi}(\sigma)\right.$; $\xi \geq 0\}$ is a uniformly continuous semigroup of operators on $H_{\sigma}$.

Proof. By Lemma 2.2, there exists, for each $\xi \geq 0$, a unique $E_{\xi} \in \mathbb{S}(\Sigma)$ such that $(T(\xi) f)^{\wedge}=E_{\xi} \hat{f}$ for every $f \in L_{p}(G)$. To complete the proof of the first assertion of the theorem, we only need to show that for each fixed $\sigma \in$ $\Sigma$, the set $\left\{E_{\xi}(\sigma) ; \xi \geq 0\right\}$ is a semigroup of operators on $H_{\sigma}$. We have, for $f \in L_{p}(G)$ and $\xi_{1}, \xi_{2} \geq 0$,

$$
\begin{aligned}
E_{\xi_{1}+\xi_{2}}(\sigma) \hat{f}(\sigma) & =\left(T\left(\xi_{1}+\xi_{2}\right) f\right)^{\wedge}(\sigma)=\left[T\left(\xi_{1}\right)\left(T\left(\xi_{2}\right) f\right)\right]^{\wedge}(\sigma) \\
& =E_{\xi_{1}}(\sigma)\left(T\left(\xi_{2}\right) f\right)^{\wedge}(\sigma)=E_{\xi_{1}}{ }^{(\sigma) E_{\xi_{2}}}{ }{ }^{(\sigma) \hat{f}(\sigma) .}
\end{aligned}
$$

Since, by Lemma 2.1, there exists $f \in \mathscr{T}_{\sigma}(G)$ such that $\hat{f}(\sigma)=I_{\sigma}$, we have $E_{\xi_{1}+\xi_{2}}(\sigma)=E_{\xi_{1}}(\sigma) E_{\xi_{2}}(\sigma)$. Hence, $\mathscr{E}=\left\{E_{\xi}(\sigma) ; \xi \geq 0, \sigma \in \Sigma\right\}$ is a semigroup of $L_{p}(G)$-multipliers.

Suppose that $T(\xi)$ is strongly continuous and let $\sigma$ be a fixed element of $\Sigma$. By Lemma 2.1, there exists $t \in \mathfrak{T}(G)$ such that $\hat{t}(\sigma)=\mathfrak{T}_{\sigma}$. Let $\epsilon>$ 0 ; there exists $\gamma>0$ such that $\|[T(\xi)-T(0)] t\|_{p}<\epsilon$ for all $\xi$ satisfying $0<\xi<\gamma$. We have

$$
\begin{aligned}
\| E_{\xi}(\sigma)- & E_{0}(\sigma)\left\|_{B\left(H_{\sigma}\right)}=\right\| E_{\xi}(\sigma)-E_{0}(\sigma) \|_{\phi_{\infty}} \quad[1, \mathrm{D} .42] \\
& =\left\|\left[E_{\xi}(\sigma)-E_{0}(\sigma)\right] \hat{t}(\sigma)\right\|_{\phi_{\infty}}=\left\|([T(\xi)-T(0)] t)^{\wedge}(\sigma)\right\|_{\phi_{\infty}} \\
& \leq\left\|([T(\xi)-T(0)] t)^{\wedge}\right\|_{\infty} \quad[1,28.34] \\
& \leq\|[T(\xi)-T(0)] t\|_{1} \quad[1,28.36] \\
& \leq\|[T(\xi)-T(0)] t\|_{p}<\epsilon
\end{aligned}
$$


for all $\xi$ satisfying $0<\xi<\gamma$. This concludes the proof.

3.2. Corollary. If $\mathcal{T}$ is strongly continuous, then there exist a subset $\Sigma_{0}$ of $\Sigma$ and an $A \in(\Sigma)(\Sigma)$ such that

$$
E_{\xi}(\sigma)= \begin{cases}E_{0}(\sigma) \exp \left(\xi A_{\sigma}\right) & \text { if } \sigma \in \Sigma_{0}, \\ 0 & \text { if } \sigma \notin \Sigma_{0} .\end{cases}
$$

Proof. By Theorem 9.6.1 of [2], $E_{0}(\sigma)$ is, for each $\sigma$, a projection operator and

$$
E_{\xi}(\sigma)=E_{\xi}(\sigma) E_{0}(\sigma)=E_{0}(\sigma) E_{\xi}(\sigma) .
$$

In particular, if $E_{0}(\sigma)=0$, then $E_{\xi}(\sigma)=0$ for all $\xi$. If for a given $\sigma, E_{0}(\sigma)$ is not the zero operator, then there exists a (unique) $A_{\sigma} \in B\left(H_{\sigma}\right)$ such that $E_{\xi}(\sigma)=E_{0}(\sigma) \exp \left(\xi A_{\sigma}\right)$. Now define $A \in \mathcal{S}(\Sigma)$ by setting $A(\sigma)=A_{\sigma}$ for each $\sigma \in \Sigma$, and set $\Sigma_{0}=\left[\sigma \in \Sigma: E_{0}(\sigma) \neq 0\right]$.

3.3. Theorem. Let $\mathcal{E}=\left\{E_{\xi}(\sigma) ; \xi \geq 0, \sigma \in \Sigma\right\}$ be a semigroup of $L_{p}(G)$ multipliers. Then $\mathcal{E}$ defines a semigroup $\mathcal{T}=\{T(\xi) ; \xi \geq 0\}$ of bounded lin. ear operators on $L_{p}(G)$, each of which commutes with right translations. If, in addition, for each $\sigma$, the set $\left\{E_{\xi}(\sigma) ; \xi \geq 0\right\}$ is a uniformly continuous semigroup of operators on $H_{\sigma}$, then $\mathcal{T}$ is a strongly continuous semigroup of operators on $L_{p}(G)$.

Proof. For each $\xi \geq 0$, we define $T(\xi)$ on $L_{p}(G)$ by $(T(\xi) f)^{\wedge}=E_{\xi} \hat{f}$, $f \in L_{p}(G)$. Then, by $[1,35.2], T(\xi)$ is a bounded linear operator on $L_{p}(G)$. That the operators $T(\xi)$ have the semigroup property follows directly from the definition. We show that $T(\xi)$ commutes with right translations. First, we note that if $f \in L_{p}(G)$, then, for each $x \in G$,

$$
\hat{f}_{x}(\sigma)=\hat{f}(\sigma) \bar{U}_{x}^{(\sigma)}
$$

for each $\sigma \in \Sigma$. In fact, for all $\xi, \eta \in H_{\sigma}$,

$$
\begin{aligned}
\left\langle\hat{f}(\sigma) \bar{U}^{(\sigma)} \xi, \eta\right\rangle=\left\langle\hat{f}(\sigma)\left(\bar{U}_{x^{-1}}^{(\sigma)} \xi\right), \eta\right\rangle & \\
= & \int_{G}\left\langle\bar{U}_{y}^{(\sigma)}\left(\bar{U}_{x^{-1}}^{(\sigma)} \xi\right), \eta\right\rangle f(y) d \lambda(y)=\int_{G}\left\langle\bar{U}_{y x^{-1}}^{(\sigma)} \xi, \eta\right\rangle f(y) d \lambda(y) \\
= & \int_{G}\left\langle\bar{U}_{y}^{(\sigma)} \xi, \eta\right\rangle_{x}(y) d \lambda(y)=\left\langle\hat{f}_{x}(\sigma) \xi, \eta\right\rangle,
\end{aligned}
$$

and hence $\hat{f}_{x}(\sigma)=\hat{f}(\sigma) U_{x^{-1}}^{(\sigma)}, \sigma \in \Sigma$.

Now $\left(T(\xi) f_{x} \hat{)}(\sigma)=\stackrel{x}{E}_{\xi}(\sigma) \hat{f}_{x}(\sigma)\right.$ and

$$
\begin{aligned}
\left([T(\xi) f]_{x}\right)^{\wedge}(\sigma) & =(T(\xi) f)^{\wedge}(\sigma) \bar{U}_{x-1}^{(\sigma)} \quad(\text { by }(1)) \\
& =E_{\xi}(\sigma) \hat{f}(\sigma) \bar{U}_{x-1}^{(\sigma)}=E_{\xi}(\sigma) \hat{f}_{x}(\sigma) \quad \text { (again by (1)). }
\end{aligned}
$$


We therefore have $\left(T(\xi) f_{x} \hat{)}(\sigma)=\left([T(\xi) f]_{x} \hat{)}(\sigma)\right.\right.$ for all $\sigma \in \Sigma$, which implies that $T(\xi) f_{x}=(T(\xi) f)_{x}$ for each $x \in G$.

Suppose now that for each $\sigma \in \Sigma$, the set $\left\{E_{\xi}(\sigma) ; \xi \geq 0\right\}$ is a uniformly continuous semigroup of operators on $H_{\sigma}$. To show that $\{T(\xi) ; \xi \geq 0\}$ is strongly continuous, we shall first show that for every coordinate function $u,\left\|\left[T(\xi)-T\left(\xi_{0}\right)\right] u\right\|_{p} \rightarrow 0$ as $\xi \rightarrow \xi_{0}$. Let $\sigma$ be an arbitrary, but fixed, element of $\Sigma$. Let $U^{(\sigma)} \in \sigma$ and let $\left\{\xi_{1}, \xi_{2}, \cdots \xi_{d_{\sigma}}\right\}$ be a basis in $H_{\sigma}$. We consider the coordinate function $u_{j k}^{(\sigma)}$ defined on $G$ by $u_{j k}^{(\sigma)}(x)=$ $\left\langle U_{x}^{(\sigma)} \xi_{k}, \xi_{j}\right\rangle$, where $j, k$ is a fixed pair from $\left\{1,2, \cdots, d_{\sigma}\right\}$. We have, for all $\sigma^{\prime} \in \Sigma$,

$$
\begin{aligned}
& \left(\left[T(\xi)-T\left(\xi_{0}\right)\right] u_{j k}^{(\sigma)}\right)^{\wedge}\left(\sigma^{\prime}\right)=\left(E_{\xi}-E_{\xi_{0}}\right)\left(\sigma^{\prime}\right) \hat{u}_{j k}^{(\sigma)}\left(\sigma^{\prime}\right) \\
& = \begin{cases}\left(E_{\xi}-E_{\xi_{0}}\right)(\sigma) u_{j k}^{(\sigma)}(\sigma) & \text { if } \sigma^{\prime}=\sigma, \\
0 & \text { if } \sigma^{\prime} \neq \sigma,\end{cases}
\end{aligned}
$$

by $[1, \mathrm{p} .80,(2)]$. Thus $\left(\left[T(\xi)-T\left(\xi_{0}\right)\right] u_{j k}^{(\sigma)}\right)^{\wedge} \epsilon \mathcal{S}_{00}(\Sigma)$ and hence

$$
\left[T(\xi)-T\left(\xi_{0}\right)\right] u_{j k}^{(\sigma)}
$$

is a trigonometric polynomial $[1,28.39]$. We now have

$$
\begin{aligned}
\left\|\left[T(\xi)-T\left(\xi_{0}\right)\right] u_{j k}^{(\sigma)}\right\|_{p} & \leq\left\|\left[T(\xi)-T\left(\xi_{0}\right)\right] u_{j k}^{(\sigma)}\right\|_{u} \\
& \leq\left\|\left[T(\xi)-T\left(\xi_{0}\right)\right] u_{j k}^{(\sigma)}\right\|_{A(G)} \quad[1,34.6] \\
& =\sum_{\sigma^{\prime} \in \Sigma} d_{\sigma^{\prime}}\left\|\left(\left[T(\xi)-T\left(\xi_{0}\right)\right] u_{j k}^{(\sigma)}\right)^{\wedge}\left(\sigma^{\prime}\right)\right\|_{\phi_{1}} \quad[1,34.4] \\
& =d_{\sigma}\left\|\left(\left[T(\xi)-T\left(\xi_{0}\right)\right] u_{j k}^{(\sigma)}\right)^{\wedge}(\sigma)\right\|_{\phi_{1}} \\
& =d_{\sigma}\left\|\left[E_{\xi}(\sigma)-E_{\xi_{0}}(\sigma)\right] \hat{u}_{j k}^{(\sigma)}(\sigma)\right\|_{\phi_{1}} \\
& \leq d_{\sigma}\left\|E_{\xi}(\sigma)-E_{\xi_{0}}(\sigma)\right\|_{\phi_{\infty}} \cdot\left\|\hat{u}_{j k}(\sigma)\right\|_{\phi_{1}} \quad[1, \text { D.52] } \\
& =d_{\sigma}\left\|E_{\xi}(\sigma)-E_{\xi_{0}}(\sigma)\right\|_{B\left(H_{\sigma}\right)} \cdot\left\|\hat{u}_{j k}^{(\sigma)}(\sigma)\right\|_{\phi_{1}} \quad[1, \text { D.4 } \\
& \rightarrow 0 \quad \text { as } \xi \rightarrow \xi_{0},
\end{aligned}
$$

by the uniform continuity of $E_{\xi}(\sigma)$. Hence, $\left\|\left[T(\xi)-T\left(\xi_{0}\right)\right] u\right\|_{p} \rightarrow 0$ as $\xi \rightarrow \xi_{0}$ for every coordinate function $u$. By the linearity of the operators $T(\xi)$,

$$
\left\|\left[T(\xi)-T\left(\xi_{0}\right)\right] t\right\|_{p} \rightarrow 0 \text { as } \xi \rightarrow \xi_{0}
$$

for every function $t \in \mathfrak{T}(G)$. That $\left\|\left[T(\xi)-T\left(\xi_{0}\right)\right] f\right\|_{p} \rightarrow 0$ as $\xi \rightarrow \xi_{0}$ for every $f \epsilon$ 
$L_{p}(G)$ now follows from the last assertion, the fact that $\mathscr{T}(G)$ is dense in $L_{p}(G)$ and the continuity of the operators $T(\xi)$. This concludes the proof.

3.4. Let $\mathcal{E}$ be as in Theorem 3.3 and suppose that, for each $\sigma \in \Sigma$, the set $\left\{E_{\xi}(\sigma) ; \xi \geq 0\right\}$ is a uniformly continuous semigroup of operators on $H_{\sigma}$. Then there exist a subset $\Sigma_{0}$ of $\Sigma$ and an $A \in \mathbb{S}(\Sigma)$ such that

$$
E_{\xi}(\sigma)= \begin{cases}E_{0}(\sigma) \exp \left(\xi_{\sigma}\right) & \text { if } \sigma \in \Sigma_{0}, \\ 0 & \text { if } \sigma \notin \Sigma_{0} .\end{cases}
$$

Let $\mathbb{Q}_{0}$ denote the infinitesimal operator of the semigroup $\mathcal{T}$ generated by $\mathcal{E}$. The following theorem gives some information about the relation between $\mathbb{Q}_{0}$ and $A$. Here, as is usual, we set $E_{0}(\sigma)=I_{\sigma}$.

3.5. Theorem. For each $f$ in the domain $D\left(\mathbb{Q}_{0}\right)$ of $\mathbb{Q}_{0}$ and $\sigma \notin \Sigma_{0}$, we have $\hat{f}(\sigma)=0$. Furthermore, $\left(\mathbb{Q}_{0} f\right)^{\wedge}=A \hat{f}$ for $f \in D\left(\mathbb{Q}_{0}\right)$; i.e. $A$ is a $\left(D\left(\mathbb{A}_{0}\right), L_{p}(G)\right)$-multiplier. If, in particular, $\mathcal{T}$ is of class $(A)$ with infinites. imal generator $\mathbb{P}$, then $\Sigma_{0}=\Sigma$. If, in addition, $A \in \mathcal{S}_{\infty}(\Sigma)$, then

$$
D(\mathbb{P})=\left[f \in L_{p}(G): A \hat{f} \in L_{p}(G)^{\wedge}\right],
$$

and $\left(\mathfrak{Q}_{f}\right)^{\hat{n}}=A \hat{f}$ for $f \in D(\mathfrak{Q})$, so that $A$ is a $\left(D(\mathfrak{Q}), L_{p}(G)\right)$-multiplier.

Proof. Let $\epsilon>0$; then there exists $y>0$ such that

$$
\left\|\mathscr{Q}_{0} f-[T(\eta) f-f] / \eta\right\|_{p}<\epsilon
$$

for $0<\eta<\gamma$. This implies that if $\sigma \notin \Sigma_{0}$, then $\hat{f}(\sigma)=0$, and if $\sigma \in \Sigma_{0}$,

$$
\left(\mathfrak{Q}_{0} f\right)^{\wedge}(\sigma)=A_{\sigma} \hat{f}(\sigma), \quad f \in D\left(\mathfrak{Q}_{0}\right) .
$$

Let $\mathcal{T}$ be of class $(A)$. Then $D\left(\mathbb{Q}_{0}\right) \subset D(\mathbb{Q})$ is dense in $L_{p}(G)$. Suppose there exists $\sigma_{0} \in \Sigma$ such that $\sigma_{0} \notin \Sigma_{0}$ and choose $f \in L_{p}(G)$ such that $\hat{f}\left(\sigma_{0}\right) \neq 0$. Given $\epsilon>0$, there exists $f^{\prime} \in D\left(\mathbb{Q}_{0}\right)$ such that $\left\|f-f^{\prime}\right\|_{p}<\epsilon$. Then

$$
\left\|\hat{f}^{\prime}\left(\sigma_{0}\right)-\hat{f}\left(\sigma_{0}\right)\right\|_{B\left(H_{\sigma_{0}}\right)} \leq\left\|f^{\prime}-f\right\|_{p}<\epsilon,
$$

which, by the first part of the theorem, implies that $f\left(\sigma_{0}\right)=0$ a contradiction. This proves that $\Sigma_{0}=\Sigma$.

To prove the last assertion of the theorem, let $\omega_{0}$ be the type of the semigroup $\mathfrak{T}$ and set $\mathfrak{L}_{0}=\bigcup\left\{T(\xi)\left[L_{p}(G)\right] ; \xi \geq 0\right\}$. For $\lambda$ with $\operatorname{Re}(\lambda)>\omega_{0}$, let $R(\lambda ; \mathbb{Q})$ denote the resolvent of $\mathbb{Q}$. Then $[2, \mathrm{p} .342]$ there exists $\omega_{1}>$ $\omega_{0}$ such that

$$
R(\lambda ; \mathfrak{A}) f=\int_{0}^{\infty} e^{-\lambda \xi} T(\xi) f d \xi, \quad f \in \mathfrak{I}_{0}, \operatorname{Re}(\lambda)>\omega_{1} .
$$

For each $\sigma \in \Sigma$, write $S_{\sigma}(f)=\hat{f}(\sigma), f \in L_{p}(G)$. Then $S_{\sigma}$ is a bounded linear 
transformation on $L_{p}(G)$ into $B\left(H_{\sigma}\right)$, and for all $f \in \mathscr{L}_{0}$,

$$
\begin{aligned}
& S_{\sigma}(R(\lambda ; \mathbb{A}) f)=\int_{0}^{\infty} e^{-\lambda \xi} S_{\sigma}(T(\xi) f) d \xi=\int_{0}^{\infty} e^{-\lambda \xi} E_{\xi}(\sigma) \hat{f}(\sigma) d \xi \\
& \quad=\int_{0}^{\infty} e^{-\lambda I_{\sigma} \xi} e^{\xi A_{\sigma}} \hat{f}(\sigma) d \xi=\int_{0}^{\infty} e^{\xi\left(A_{\sigma}-\lambda I_{\sigma}\right)} d \xi \hat{f}(\sigma)=\left(\lambda I_{\sigma}-A_{\sigma}\right)^{-1} \hat{f}(\sigma),
\end{aligned}
$$

for all $\lambda$ with $\operatorname{Re}(\lambda)>\max \left(\omega_{1},\|A\|_{\infty}\right),[2,(11.2 .3)]$. Since $\varrho_{0}$ is dense in $L_{p}(G),[2, \mathrm{p} .342]$, we have

$$
(R(\lambda ; \mathfrak{Q}) f)^{\wedge}(\sigma)=\left(\lambda I_{\sigma}-A_{\sigma}\right)^{-1} \hat{f}(\sigma)
$$

for all $f \in L_{p}(G), \operatorname{Re}(\lambda)>\max \left(\omega_{1},\|A\|_{\infty}\right)$. We now make use of the last assertion to prove that

$$
D(\mathfrak{Q})=\left[f \in L_{p}(G): A \hat{f} \in L_{p}(G)^{\wedge}\right]
$$

Let $f \in D(\mathscr{Q})$ and let $\sigma$ be an arbitrary element of $\Sigma$. Choose $\lambda$ such that $\lambda>\max \left(\omega_{1},\|A\|_{\infty}\right)$. Then there exists $g \in L_{p}(G)$ such that $f=R(\lambda ; \mathbb{A}) g$, and we have

$$
\begin{aligned}
(\mathfrak{Q} f)^{\wedge}(\sigma) & =[\lambda R(\lambda, \mathfrak{Q}) g-g]^{\wedge}(\sigma)=\lambda\left(\lambda I_{\sigma}-A_{\sigma}\right)^{-1} \hat{g}(\sigma)-\hat{g}(\sigma) \\
& =A_{\sigma}\left(\lambda I_{\sigma}-A_{\sigma}\right)^{-1} \hat{g}(\sigma)=A_{\sigma} \hat{f}(\sigma) .
\end{aligned}
$$

Since $\sigma$ was arbitrary, $\left(\mathfrak{l} f \hat{)}(\sigma)=A_{\sigma} \hat{f}(\sigma)\right.$ for every $\sigma \in \Sigma$. Thus, if $f \in D(\mathbb{A})$,

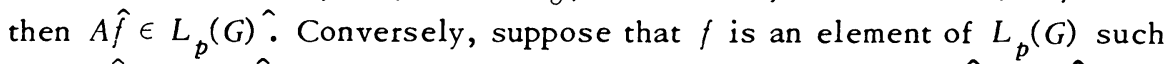

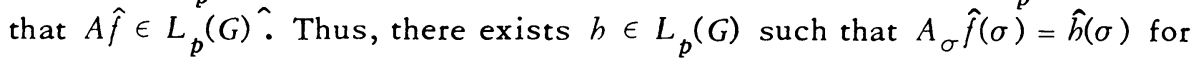
all $\sigma \in \Sigma$. The function $g=\lambda f-h \in L_{p}(G)$ for all complex numbers $\lambda$. If $\lambda>\max \left(\omega_{1},\|A\|_{\infty}\right)$, we have

$$
\begin{aligned}
(R(\lambda ; \mathfrak{Q}) g)^{\wedge}(\sigma) & =\left(\lambda I_{\sigma}-A_{\sigma}\right)^{-1} \hat{g}(\sigma) \\
& =\left(\lambda I_{\sigma}-A_{\sigma}\right)^{-1}\left(\lambda \hat{f}(\sigma)-A_{\sigma} \hat{f}(\sigma)\right)=\hat{f}(\sigma),
\end{aligned}
$$

for all $\sigma \in \Sigma$. Hence $R(\lambda ; \mathscr{Q}) g=f$, which implies that $f \in D(\mathscr{Q})$. This concludes the proof.

3.6. Remarks. As an example of the situation described in Theorem 3.1, we mention the heat-diffusion semigroup $\left\{T^{t} ; t \geq 0\right\}$ of operators on $L_{p}(G)$ for a compact Lie group $G$ discussed by Stein [4, p. 38]. Also, one obtains an illustration of Theorem 3.3 by considering the Fourier-Stielties transforms of the semigroup $\left\{\mu_{t} ; t \geq 0\right\}$ of measures in $M(G)$ studied by Hunt [3].

\section{REFERENCES}

1. E. Hewitt and K. A. Ross, Abstract harmonic analysis. Vol. II: Structure and analysis for compact groups. Analysis on locally compact Abelian groups, Die Grundlehren der math. Wissenschaften, Band 152, Springer-Verlag, Berlin and New York, 1970. MR $41 \# 7378$; erratum, 42, p. 1825. 
2. E. Hille and R. S. Phillips, Functional analysis and semi-groups, rev. ed., Amer. Math. Soc. Colloq. Publ., vol. 31, Amer. Math. Soc., Providence, R. I., 1957. MR 19, 664 .

3. G. A. Hunt, Semi-groups of measures on Lie groups, Trans. Amer. Math. Soc. 81 (1956), 264-293. MR 18, 54.

4. E. M. Stein, Topics in harmonic analysis related to the Littlewood-Paley theory, Ann. of Math. Studies, no. 63, Princeton Univ. Press, Princeton, N. J.; Univ. of Tokyo Press, Tokyo, 1970. MR 40 \#6176.

5. V. A. Babalola and A. Olubummo, Semigroups of operators commuting with translations, Colloq. Math. 31 (1974), 241-246.

DEPARTMENT OF MATHEMATICS, UNIVERSITY OF IBADAN, IBADAN, NIGERIA 